\title{
THE WIENER LEMMA AND CERTAIN OF ITS GENERALIZATIONS
}

\section{THIERRY COQUAND AND GABRIEL STOLZENBERG}

Let $\Gamma$ be the unit circle in the complex plane. Let $\mathscr{A}$ be the Banach algebra of all complex valued continuous functions on $\Gamma$ with absolutely convergent Fourier series.

Norbert Wiener [10] proved that if any $f$ in $\mathscr{A}$ is invertible in the ring of continuous functions on $\Gamma$, then $1 / f$ also is an element of $\mathscr{A}$. Paul Lévy [7] generalized Wiener's result, showing that for each $f$ in $\mathscr{A}$ and each complex analytic function $\Phi$ that is defined on a neighborhood of $f(\Gamma), \Phi(f)$ belongs to $\mathscr{A}$.

Lévy did so by an argument that shows, more generally, that $\Phi(f)$ belongs to $\mathscr{A}$ whenever $f$ is in $\mathscr{A}^{d}$ and $\Phi$ is analytic on some neighborhood of $f(\Gamma)$ in $\mathbf{C}^{d}$. Later, G. E. Šilov [9] established such a result for a class of Banach algebras of continuous functions that includes $\mathscr{A}$. Silov's proof uses the Cauchy-Weil integral formula for an analytic function of several complex variables.

\section{AN INTEGRAL FORMULA}

Here, we prove the several variable form of the Wiener-Lévy theorem by showing that for each $f$ in $\mathscr{A}^{d}$ and each $\Phi$ analytic on a neighborhood of $f(\Gamma)$ in $\mathbf{C}^{d}, \Phi(f)$ is given by a one variable Cauchy integral formula for a related $\mathscr{A}$-valued function that is analytic on a neighborhood of $\Gamma$ in the plane.

Let $u$ be the identity function restricted to $\Gamma$, viewed as an element of $\mathscr{A}$. If $z$ is at a positive distance from $\Gamma$, either $1 / z$ times the geometric series in $u / z$ or $-1 / u$ times the geometric series in $z / u$ is an inverse for $z-u$ in $\mathscr{A}$. Therefore, as an $\mathscr{A}$-valued function of $z, 1 /(z-u)$ is uniformly continuous on the complement of each neighborhood of $\Gamma$.

For each $n$, let $f_{n}$ be the mapping from $\Gamma$ to $\mathbf{C}^{d}$ each coordinate of which is the truncation from $-n$ to $n$ of the Fourier series

Received by the editors March 12, 1990.

1980 Mathematics Subject Classification (1985 Revision). Primary 42A28. 
of the corresponding coordinate of $f$. Then $f_{n}$ is an element of $\mathscr{A}^{d}$. Let $f_{n}(z)$ be the induced mapping from the punctured plane to $C^{d}$ whose coordinates are the polynomials in $z$ and $1 / z$ gotten by substituting $z$ for $u$.

View $\mathbf{C}^{d}$ as a subspace of $\mathscr{A}^{d}$ by regarding $\mathbf{C}$ as a subalgebra of $\mathscr{A}$. Use the local power series expansions for $\Phi$ to extend it to be an $\mathscr{A}$-valued analytic function on a neighborhood of $f(\Gamma)$ in $\mathscr{A}^{d}$.

For each $f$ and $n, f-f_{n}+f_{n}(z)$ maps the punctured plane into $\mathscr{A}^{d}$, and for $n$ large enough and $r<1<R$ sufficiently close to 1 , it maps a planar neighborhood of $\{z: r \leq|z| \leq R\}$ into the domain of $\Phi$ in $\mathscr{A}^{d}$. Therefore, if $\Gamma(R, r)$ denotes the oriented boundary of this annulus,

$$
I=\frac{1}{2 \pi i} \int_{\Gamma(R, r)} \frac{\Phi\left(f-f_{n}+f_{n}(z)\right)}{z-u} d z
$$

is an element of $\mathscr{A}$.

Because convergence in $\mathscr{A}$ implies uniform convergence on $\Gamma$, the value of $I$ at each $p$ in $\Gamma$ is

$$
\frac{1}{2 \pi i} \int_{\Gamma(R, r)} \frac{\Phi\left(f(p)-f_{n}(p)+f_{n}(z)\right)}{z-p} d z .
$$

For each $p$, the numerator of the integrand is an analytic function of $z$ on a neighborhood of $\{z: r \leq|z| \leq R\}$, and the expression above is a Cauchy integral formula for its value at $z=p$. But that value is $\Phi\left(f(p)-f_{n}(p)+f_{n}(p)\right)=\Phi(f(p))$. Hence, $I$ is an element of $\mathscr{A}$ that, as a function on $\Gamma$, is equal to $\Phi(f)$.

\section{A. P. CALDERón's INTEGRAl FORMUla}

If $h$ is any complex valued analytic function on a neighborhood of $\Gamma$, its Fourier series is the restriction to $\Gamma$ of its Laurent expansion on some $\{z: 1 / R \leq|z| \leq R\}$. Cauchy's estimate shows immediately that $h$ is in $\mathscr{A}$ and that $\|h\|_{\mathscr{A}}<M /(R-1)$, where $M$ is any bound for $h$ on $\{z: 1 / R \leq|z| \leq R\}$.

Maintaining the notation of the previous section, it follows that for $\epsilon>0$ sufficiently small and $n \equiv n(\epsilon)$ sufficiently large, if $\partial(\epsilon)$ is the $d$-fold product of the circle of radius $\epsilon$ about 0 in 
C, then

$$
J=(2 \pi i)^{-d} \int_{\partial(\epsilon)} \frac{\Phi\left(f_{n}+w\right)}{\Pi\left(w+f_{n}-f\right)} d w
$$

defines an element of $\mathscr{A}$. Here $\Pi$ is the product mapping from $\mathscr{A}^{d}$ to $\mathscr{A}$. If for each $p$ in $\Gamma$, we set $\partial_{n}(p, \epsilon) \equiv f_{n}(p)+\partial(\epsilon)$ and $v \equiv f_{n}(p)+w$, we can reason as above to get

$$
J(p)=(2 \pi i)^{-d} \int_{\partial_{n}(p, \epsilon)} \frac{\Phi(v)}{\Pi(v-f(p))} d v .
$$

But the expression above is a $d$-dimensional Cauchy integral formula for the value of $\Phi(v)$ at $v=f(p)$ in $\mathbf{C}^{d}$. Hence $J$ is an element of $\mathscr{A}$ that as a function on $\Gamma$ is equal to $\Phi(f)$.

This lovely and illuminating idea was discovered more than forty years ago by A. P. Calderón. For the case $d=1$, and with the functions of class $C^{2}$ given the role played here by the ones that are analytic in a neighborhood of $\Gamma$, it can be found on $p$. 246 of volume I of the 1959 edition of A. Zygmund's treatise, Trigonometric series [12].

\section{A DIVISION LEMMA}

Let $\mathscr{B}$ be the Banach algebra of all complex-valued continuous functions on $\mathbf{R}$ that are Fourier transforms of elements of $L^{1}(\mathbf{R})$. The $\mathscr{B}$ norm of any such function is taken to be the $L^{1}(\mathbf{R})$ norm of the element of which it is the transform.

If $f$ is the Fourier transform of $F$, we let $f_{n}$ denote the Fourier transform of the product of $F$ with the characteristic function of $[-n, n]$. Thus $f_{n}$ is a sequence of entire functions whose restrictions to $\mathbf{R}$ converge in $\mathscr{B}$ to $f$. Because the $\mathscr{B}$-norm dominates the sup norm over $\mathbf{R}, f_{n}$ also converges uniformly to $f$ on $\mathbf{R}$.

Wiener used his lemma about the group of units of $\mathscr{A}$ to prove a certain division lemma for $\mathscr{B}$. However, here we depart from Wiener's approach and show instead how each of the two integral formulas for the units lemma for $\mathscr{A}$ can be modified to get similar ones for the division lemma for $\mathscr{B}$. The constructions that we give work also for $\mathscr{A}$.

Division lemma. If $f$ and $g$ are in $\mathscr{B}$, and $f$ is bounded away from zero on the support of $g$, then $g / f$ is in $\mathscr{B}$. 
Proof. Because functions in $\mathscr{B}$ vanish at infinity, the lower bound for $|f|$ on the support of $g$ determines a finite interval containing that support.

Two integrations by parts and the Fourier inversion theorem show that $\mathscr{B}$ contains every $C^{2}$ function with compact support. Let $\delta$ be such a function that is 1 on the support of $g$ and on whose support $f$ is bounded away from 0 . Then $g / f=g(\delta / f)$. So we may assume that $g$ is of class $C^{2}$.

In that case, if $|w| \equiv \epsilon>0$ is so small and $n \equiv n(\epsilon)$ is so large that $f_{n}+w$ is bounded away from zero on the support of $g$, then two integrations by parts show that $\phi_{n}(w) \equiv g /\left(f_{n}+w\right)$ maps $\Gamma(\epsilon) \equiv\{w \in \mathbf{C}:|w|=\epsilon\}$ continuously to $\mathscr{B}$.

If $n$ also is taken so large that $\left\|f-f_{n}\right\|_{\mathscr{B}}<\epsilon$, then the sums of the positive powers of $\left(f-f_{n}\right) / w$ converge uniformly on $\Gamma(\epsilon)$ to an element of $\mathscr{B}$ that equals $1 /\left(w+f_{n}-f\right)-1 / w$ as a function on $\mathbf{R}$. Therefore, the Calderón type integral

$$
\frac{1}{2 \pi i} \int_{\Gamma(\epsilon)} \frac{\phi_{n}(w) d w}{w+f_{n}-f}
$$

defines an element $h$ of $\mathscr{B}$. Because convergence in $\mathscr{B}$ implies uniform convergence on $\mathbf{R}, h(p)$ vanishes off the support of $g$ and on it is given by a Cauchy integral formula for the value of $\Phi(v) \equiv g(p) / v$ at $f(p)$. Hence, $h=g / f$ on $\mathbf{R}$.

Second proof. We may assume that $g$ is of class $C^{2}$ and supported on a finite union of intervals $\beta$ on which $f$ is bounded away from 0 . Two integrations by parts show that if $v$ is the identity function on $\mathbf{R}$, then $g /(z-v)$ is uniformly continuous as a $\mathscr{B}$-valued function of $z$ on the complement of each neighborhood of $\beta$ in $\mathbf{C}$.

For $n$ sufficiently large and $\epsilon \equiv \epsilon(n)>0$ sufficiently small, the sums of the positive powers of $\left(f-f_{n}\right) / f_{n}(z)$ converge uniformly on the $\epsilon$-neighborhood of $\beta$ to an element of $\mathscr{B}$ that equals $1 /\left(f-f_{n}+f_{n}(z)\right)-1 / f_{n}(z)$ as a function on $\mathbf{R}$.

Therefore, if $\partial$ is the oriented boundary of any sufficiently small neighborhood of $\beta$, then by a familiar line of reasoning,

$$
\frac{1}{2 \pi i} \int_{\partial} \frac{1}{f-f_{n}+f_{n}(z)} \frac{g d z}{z-v}
$$

is an element of $\mathscr{B}$ that, as a function on $\mathbf{R}$, is equal to $g / f$. 


\section{WIENER's GENERAL TAUBERIAN THEOREM [10]}

Theorem. If $b$ is any bounded linear functional on $L^{1}(\mathbf{R})$, its convolution with every member of $L^{1}(\mathbf{R})$ vanishes at $+\infty$ provided that it does so for at least one member whose Fourier transform has a continuous inverse on $\mathbf{R}$.

Proof. If $K$ is in $L^{1}(\mathbf{R})$ and $b * K$ vanishes at $+\infty$, then so does the convolution of $b * K$ with each $F$ in $L^{1}(\mathbf{R})$. But $(b * K) * F=$ $b *(K * F)$, and the set of $G$ such that $b * G$ vanishes at $+\infty$ is closed in $L^{1}(\mathbf{R})$. Hence, if $b * K$ vanishes at $+\infty$, this condition persists when $K$ is replaced by anything belonging to the closure of $\left\{K * F: F \in L^{1}(\mathbf{R})\right\}$.

By the division lemma, if $\widehat{K}$ has a continuous inverse on $\mathbf{R}$ and $\widehat{G}$ has compact support, then $\widehat{G} / \widehat{K}$ is the Fourier transform of some element $F$ of $L^{1}(\mathbf{R})$. Hence, by the inversion theorem, $G=K * F$. Because every element of $L^{1}(\mathbf{R})$ is a limit of ones whose Fourier transform has compact support, the argument of the preceding paragraph establishes the desired result.

\section{WIENER'S PROOF OF THE UNITS LEMMA}

Let us compare Wiener's own proof of the units lemma, and Lévy's generalization of it, with the ones given by the integral formulas of $\S \S 1$ and 2. The heart of it begins with a demonstration that for a certain one parameter family of functions $U_{r}$ in $\mathscr{A}$, each of which is constantly 1 on some neighborhood of 1 , if for each $f$ in $\mathscr{A}$ and $p$ in $\Gamma$, we set $f_{p}(x) \equiv f(p x)$, then $\left(f_{p}-f(p)\right) U_{r} \rightarrow 0$ in $\mathscr{A}$ as $r \rightarrow \infty$, uniformly for $p$ in $\Gamma$.

It follows from this that if $|f(p)|>0$ and $r$ is large enough, then the geometric series in $\left(1-f_{p} / f(p)\right) U_{r}$ is an inverse for $f(p)+\left(f_{p}-f(p)\right) U_{r}$ in $\mathscr{A}$. Hence, because $f_{p}$ and $f(p)+$ $\left(f_{p}-f(p)\right) U_{r}$ agree near 1 , the composition of this geometric series with $x \rightarrow x / p$ is an element $h$ of $\mathscr{A}$ that equals $1 / f$ on a neighborhood of $p$.

Therefore, each subset $S$ of $\Gamma$ on which $f$ is bounded away from 0 can be covered by finitely many arcs on each of which $f$ is continuously invertible by an element of $\mathscr{A}$. Thus, with the aid of any partition of unity in $\mathscr{A}$ that is subordinate to these arcs-say, by functions of class $C^{2}$, we can construct an element of $\mathscr{A}$ that is a continuous inverse to $f$ on all of $S$. So, if $f$ is bounded away from 0 on $\Gamma$, then $1 / f$ is an element of $\mathscr{A}$. 
Paul Lévy observed that the same line of reasoning works for any analytic function $\Phi(w)$ in place of $1 / w$, because all that we need are the local power series expansions about points of the form $f(p)$. For the same reason, the argument works for any $f$ in $\mathscr{A}^{d}$ and $\Phi$ analytic on a neighborhood of $f(\Gamma)$ in $\mathbf{C}^{d}$.

Wiener chose $U_{r}$ to be a one parameter family of trapezoidal functions-viewing a function on $\mathbf{R}$ supported on a closed subinterval of $(-\pi, \pi)$ as a function on $\Gamma$ that vanishes near $i$, and showing that for functions supported on any one such subinterval, the $\mathscr{A}$ and $\mathscr{B}$ norms are equivalent. However, the short computation that he made to prove $\left(f_{p}-f(p)\right) U_{r} \rightarrow 0$ as $r \rightarrow \infty$ can be used to show, more generally, that for all $V$ and $g$ in $\mathscr{B}$, if $V_{r}(x) \equiv V(r x)$ and $g_{p}(x) \equiv g(p+x)$, then $\left(g_{p}-g(p)\right) V_{r}$ converges to 0 in $\mathscr{B}$, uniformly for $p$ in $\mathbf{R}$.

For another simple proof of this last assertion, note first that because $V_{r}$ and $V$ are equal in norm, it suffices to prove it for $g$ and $V$ belonging to some dense subset of $\mathscr{B}$. So we may suppose that $g$ and $V$ are Fourier transforms of compactly supported continuous functions, $G$ and $v$. In that case, it is especially easy to check that $\left\|\left(g_{p}-g(p)\right) V_{r}\right\|_{\mathscr{B}}$ is bounded above by $\iint|G(x)||v(t-x / r)-v(t)| d x d t$, and that the latter converges to 0 as $r \rightarrow \infty$.

\section{THE COMMON IDEA}

Like Wiener's proof of the units lemma, Calderón's method exploits the observation that if we view $\mathbf{C}$ as a subalgebra of $\mathscr{A}$, then the power series expansions for $\Phi(w) \equiv 1 / w$ extend it to be an $\mathscr{A}$-valued analytic function near $\mathbf{C}^{*}$ in $\mathscr{A}$. Our own method, like Lévy's, employs the generalization of this to the case that $\Phi$ is an arbitrary complex analytic function.

Each method involves a trade. Putting $\Pi\left(w+f_{n}-f\right)$ in the denominator of the integrand forces us to put $\Phi\left(f_{n}+w\right)$ in the numerator. This works only if we start out knowing a strong form of the result for $f_{n}$ in place of $f$. Putting $\Phi\left(f-f_{n}+f_{n}(z)\right)$ in the numerator requires no such prior knowledge about $f_{n}$. But it is parasitic upon the ordinary Cauchy integral formula for functions analytic on a neighborhood of $\Gamma$.

Comparing the arguments in $\S \S 1$ and 2, this may seem to be a distinction without a difference. As evidence that it is not, consider the question of showing that $\Phi(f)$ is in $\mathscr{B}$ whenever $f$ is in 
$\mathscr{B}^{d}, \Phi$ is analytic on a neighborhood of $f(\mathbf{R}) \cup\{0\}$ in $\mathbf{C}^{d}$ and $\Phi(0)=0$.

Even though $f_{n}$ is analytic on a strip about $\mathbf{R}$, we lack a counterpart to the Cauchy integral formula over $\Gamma(R, r)$, and this blocks a straightfoward application of the method of $\S 1$.

On the other hand, it easily is verified that the class $\mathscr{E}$ of compactly supported $C^{2}$ functions is dense in $\mathscr{B}$, and also that for each $g$ in $\mathscr{E}^{d}$, if $\Phi$ is analytic on a neighborhood of $g(\mathbf{R})$ in $\mathbf{C}^{d}$ and $\epsilon>0$ is sufficiently small, then $w \rightarrow \Phi(g+w)-\Phi(w)$ maps the support of $\partial(\epsilon)$ continuously to $\mathscr{B}$. Consequently, we can employ the method of $\S 2$, replacing $\mathscr{A}$ by $\mathscr{B}$ and $f_{n}$ by a suitable $g$ in $\mathscr{E}^{d}$, to show that if $\Phi(0)=0$, then $J$ is an element of $\mathscr{B}$ that, as a function on $\mathbf{R}$, is equal to $\Phi(f)$.

To see that $\mathscr{E}$ is dense in $\mathscr{B}$, observe that if $f$ is any compactly supported element of $\mathscr{B}$ and $V$ is any element of $\mathscr{E}$ for which $f=f V$, then $f_{n} V$ is a sequence in $\mathscr{E}$ that converges to $f$ in $\mathscr{B}$.

To see that for $g$ in $\mathscr{E}^{d}$ and $\epsilon>0$ sufficiently small, $\boldsymbol{\Phi}(g+w)-\boldsymbol{\Phi}(w)$ is a continuous $\mathscr{B}$-valued function of $w$ in $\partial(\epsilon)$, factor it through $\mathscr{E}$ and use two integrations by parts to verify that the $\mathscr{B}$ norm of any member of $\mathscr{E}$ is dominated by its $C^{2}$ norm times the length of any interval on which it is supported.

\section{General Settings}

The argument just given works equally well when $\mathbf{R}$ is replaced by a differentiable manifold $M$ and $\mathscr{B}$ by the completion of $C_{c}^{\infty}(M)$ in an algebra norm that dominates the sup norm but, for each compact subset of $M$ and some $k \in \mathbf{N}$, is majorized by the $C^{k}$ norm on the subalgebra of $C^{\infty}$ functions supported in that set.

Likewise, for the argument used in $\S 2$, we can replace $\Gamma$ by a compact $K \subset \mathbf{C}^{m}$ and $\mathscr{A}$ by the completion of $\mathscr{H}(K)$ in an algebra norm that dominates the sup norm on $K$ but is majorized by the sup norm on each compact neighborhood of $K$. Here, $\mathscr{H}(K)$ is the algebra of functions analytic about $K$.

Suppose $B \subset C(K)$ is the completion of $\mathscr{H}(K)$ in an algebra norm that dominates the sup norm. To prove that the $B$ norm is majorized by the sup norm on each compact neighborhood of $K$, it suffices to show how to construct, inside any such neighborhood, a compact analytic polyhedron containing $K$ and a Cauchy-Weil 
type integral whose kernel is a continuous $B$-valued function on the support of the cycle over which the integral is defined. This, or something very like it, also is what we need to construct a $B$-valued integral formula of the kind presented in $\S 1$.

\section{8. ŠILOV'S THEOREM REVISITED}

These considerations allow us to make a certain conceptual simplification in those proofs of Silov's generalization of the WienerLévy theorem that use a Cauchy-Weil integral formula. In them, even if we already have such a setup for $K$ and $B$, for each $f$ in $B^{d}$ we must pass to the graph of $f$ in $\mathbf{C}^{m+d}$, lift $B$ to an algebra on it, and create a similar environment of analytic polyhedra and Cauchy-Weil type integral formulas.

But once we have such an environment for $K$, there is no need to leave it. Given any $f$ in $B^{d}$ and $\Phi$ analytic on a neighborhood of $f(K)$ in $\mathbf{C}^{d}$, by taking a $g$ sufficiently close to $f$ in $B^{d}$, each coordinate of which is analytic on a neighborhood of $K$, and using either of the two methods presented here, we can express $\Phi(f)$ either as a $B$-valued Cauchy-Weil type integral of $\Phi(f-g+g(z))$ or as a $B$-valued Cauchy integral of $\Phi(g+w) / \Pi(g+w-f)$ over some $\partial(\epsilon)$.

To see why this sometimes may be a good thing to do, we need only contrast the treatment accorded to analytic functions of members of $\mathscr{A}$ by the methods presented here with their handling by the usual proofs of Śilov's theorem-for example, in [2] or [4].

\section{ACKNOWLEDGMENTS}

The integral formula presented in $\S 1$ was discovered during a conversation about the relative merits of Errett Bishop's and N. G. de Bruijn's very different ways of viewing Gelfand's theory of the spectrum as a computational scheme. See [1] and [3]. Ideas of each played an important role in leading us to it.

The argument at the end of $\S 5$ was constructed by reading the proof of Lemma 9.2 of [8] under the assumption that measure theory does not exist.

It would be difficult to find a more elegant proof of the WienerLévy theorem along the lines of Wiener's own than that given by Jean-Pierre Kahane in [5].

Conversations with Y. Katznelson, as well as his text [6], helped one of us to learn, and maybe even understand, some of the basic ideas of the subject to which this paper pretends to be devoted. 


\section{REFERENCES}

1. E. Bishop and D. Bridges, Constructive analysis, Springer-Verlag, Berlin and New York, 1985 (Revised ed. of Bishop's Foundations of constructive analysis, McGraw-Hill, 1967).

2. N. Bourbaki, Éléments de mathématique, théories spectrales, Hermann, Paris, 1967.

3. N. G. De Bruijn and W. Van Der Meiden, Notes on Gelfand's theory, Indag. Math. 31 (1968), 467-474.

4. L. Hormander, An introduction to complex analysis in several variables, Van Nostrand, Princeton, N.J., 1966.

5. J.-P. Kahane, Séries de Fourier absolument convergentes, Ergeb. Math. Grenzgeb., Band 50, Springer-Verlag, Berlin and New York, 1970.

6. Y. Katznelson, An introduction to harmonic analysis, Dover, 1976 (2nd corrected edition).

7. P. Lévy, Sur la convergence absolue des séries de Fourier, Proc. Composito Math. 1 (1934), 1-14.

8. W. Rudin, Functional analysis, McGraw-Hill, New York, 1973.

9. G. E. Silov, On the decomposition of a commutative normed ring into the direct sum of ideals, Mat. Sbornik 74 (1953), 353-364. (Russian)

10. N. Wiener, Tauberian theorems, Ann. of Math. (2) 33 (1932), 1-100.

11. _ The Fourier integral and certain of its applications, Cambridge Univ., 1933.

12. A. Zygmund, Trigonometric series, Cambridge Univ., 1959 (2nd edition).

Projet Formel, INRIA, 78150, Rocquencourt, France

E-mail address: coquand@inria.inria.fr

Department of Mathematics, Harvard University, Cambridge, Massachusetts 02138 and Department of Mathematics, Northeastern UNIVERSITY, Boston, MASSACHUSETTS 02115

E-mail address: gabe@zariski.harvard.edu 
